\title{
Reverses of the Jensen-Type Inequalities for Signed Measures
}

\author{
Rozarija Jakšić, Josip Pečarić, and Mirna Rodić Lipanović \\ Faculty of Textile Technology, University of Zagreb, Prilaz baruna Filipovića 28a, 10000 Zagreb, Croatia
}

Correspondence should be addressed to Rozarija Jakšić; jaksic.rozarija@gmail.com

Received 26 March 2014; Accepted 8 July 2014; Published 12 August 2014

Academic Editor: Shawn X. Wang

Copyright (C) 2014 Rozarija Jakšić et al. This is an open access article distributed under the Creative Commons Attribution License, which permits unrestricted use, distribution, and reproduction in any medium, provided the original work is properly cited.

In this paper we derive refinements of the Jensen type inequalities in the case of real Stieltjes measure $d \lambda$, not necessarily positive, which are generalizations of Jensen's inequality and its reverses for positive measures. Furthermore, we investigate the exponential and logarithmic convexity of the difference between the left-hand and the right-hand side of these inequalities and give several examples of the families of functions for which the obtained results can be applied. The outcome is a new class of Cauchy-type means.

\section{Introduction}

The authors in [1] gave some conditions on the real Stieltjes measure $d \lambda$, not necessarily positive, under which the Jensen inequality and the converse of the Jensen inequality hold for continuous convex function $\varphi$. These results are derived by using the Green function $G$ defined on $[\alpha, \beta] \times[\alpha, \beta]$ by

$$
G(t, s)= \begin{cases}\frac{(t-\beta)(s-\alpha)}{\beta-\alpha} & \text { for } \alpha \leq s \leq t, \\ \frac{(s-\beta)(t-\alpha)}{\beta-\alpha} & \text { for } t \leq s \leq \beta .\end{cases}
$$

The function $G$ is convex and continuous with respect to both $s$ and $t$.

Several interesting results concerning the Jensen type inequalities have been derived by means of the function $G$. The first one, which is stated in the following theorem, gives the conditions on the real Stieltjes measure $d \lambda$, not necessarily positive, under which the Jensen inequality holds for continuous convex function $\varphi$.

Theorem 1 (see [1]). Let $g:[a, b] \rightarrow \mathbb{R}$ be continuous function and $[\alpha, \beta]$ interval such that the image of $g$ is a subset of $[\alpha, \beta]$. Let $\lambda:[a, b] \rightarrow \mathbb{R}$ be continuous function or the function of bounded variation such that $\lambda(a) \neq \lambda(b)$ and $\int_{a}^{b} g(x) d \lambda(x) / \int_{a}^{b} d \lambda(x) \in[\alpha, \beta]$. Then the following two statements are equivalent.
(1) For every continuous convex function $\varphi:[\alpha, \beta] \rightarrow \mathbb{R}$

$$
\varphi\left(\frac{\int_{a}^{b} g(x) d \lambda(x)}{\int_{a}^{b} d \lambda(x)}\right) \leq \frac{\int_{a}^{b} \varphi(g(x)) d \lambda(x)}{\int_{a}^{b} d \lambda(x)}
$$

holds.

(2) For all $s \in[\alpha, \beta]$

$$
G\left(\frac{\int_{a}^{b} g(x) d \lambda(x)}{\int_{a}^{b} d \lambda(x)}, s\right) \leq \frac{\int_{a}^{b} G(g(x), s) d \lambda(x)}{\int_{a}^{b} d \lambda(x)}
$$

holds, where the function $G:[\alpha, \beta] \times[\alpha, \beta] \rightarrow \mathbb{R}$ is defined in (1).

Furthermore, the statements (1) and (2) are also equivalent if we change the sign of inequality in both (2) and (3). Also note that for every continuous concave function $\varphi:[\alpha, \beta] \rightarrow \mathbb{R}$ the inequality (2) is reversed.

Remark 2. Note that in the case of positive measure $d \lambda$ we get some well known results. If the function $\lambda$ is increasing and bounded with $\lambda(a) \neq \lambda(b)$, then inequality (2) becomes Jensen's integral inequality. On the other hand, if the function $g$ is continuous and monotonic, and $\lambda$ is either continuous or of bounded variation, satisfying

$$
\lambda(a) \leq \lambda(x) \leq \lambda(b), \quad \forall x \in[a, b], \lambda(a)<\lambda(b),
$$


then inequality (2) becomes the Jensen-Steffensen inequality given by Boas in [2] (see also [3], page 59). Several other theorems concerning the inequality (2) or its reverse can be found in [3].

Similar results have also been derived for the converse of the Jensen inequality. The following theorem from [1] gives the conditions on the real Stieltjes measure $d \lambda$, not necessarily positive such that $\lambda(a) \neq \lambda(b)$, under which the converse of the Jensen inequality holds for continuous convex function $\varphi$.

Theorem 3 (see [1]). Let $g:[a, b] \rightarrow \mathbb{R}$ be continuous function and $[\alpha, \beta]$ be an interval such that the image of $g$ is a subset of $[\alpha, \beta]$. Let $m, M \in[\alpha, \beta](m \neq M)$ be such that $m \leq g(t) \leq M$ for all $t \in[a, b]$. Let $\lambda:[a, b] \rightarrow \mathbb{R}$ be continuous function or the function of bounded variation, and $\lambda(a) \neq \lambda(b)$. Then the following two statements are equivalent.

(1) For every continuous convex function $\varphi:[\alpha, \beta] \rightarrow \mathbb{R}$

$$
\frac{\int_{a}^{b} \varphi(g(x)) d \lambda(x)}{\int_{a}^{b} d \lambda(x)} \leq \frac{M-\bar{g}}{M-m} \varphi(m)+\frac{\bar{g}-m}{M-m} \varphi(M)
$$

holds, where $\bar{g}=\int_{a}^{b} g(x) d \lambda(x) / \int_{a}^{b} d \lambda(x)$.

(2) For all $s \in[\alpha, \beta]$

$$
\frac{\int_{a}^{b} G(g(x), s) d \lambda(x)}{\int_{a}^{b} d \lambda(x)} \leq \frac{M-\bar{g}}{M-m} G(m, s)+\frac{\bar{g}-m}{M-m} G(M, s)
$$

holds, where the function $G:[\alpha, \beta] \times[\alpha, \beta] \rightarrow \mathbb{R}$ is defined in (1).

Furthermore, the statements (1) and (2) are also equivalent if we change the sign of inequality in both (5) and (6).

Remark 4. If we set in Theorem $3 m=\alpha$ and $M=\beta$, the inequality (6) transforms into (see also [1])

$$
\frac{\int_{a}^{b} G(g(x), s) d \lambda(x)}{\int_{a}^{b} d \lambda(x)} \leq 0
$$

In his papers $[4,5]$, Dragomir gave some inequalities concerning reverses of the Jensen inequality for positive measure. In this paper we give a generalization of those results and derive similar Jensen-type inequalities in the case of real Stieltjes measure $d \lambda$ which is not necessarily positive. Furthermore, we investigate the exponential and logarithmic convexity of the differences between the left-hand and the right-hand side of the obtained inequalities and give several examples of the families of functions for which those results can be applied.

\section{Main Results}

Throughout this paper we will use the notation

$$
\bar{g}=\frac{\int_{a}^{b} g(x) d \lambda(x)}{\int_{a}^{b} d \lambda(x)} .
$$

The following result holds.

Theorem 5. Let $g:[a, b] \rightarrow \mathbb{R}$ be a continuous function and $[\alpha, \beta]$ an interval. Let $m, M \in\langle\alpha, \beta\rangle$ be such that $m \neq M$ and $m \leq g(t) \leq M$ for all $t \in[a, b]$. Let $\lambda:[a, b] \rightarrow \mathbb{R}$ be a continuous function or a function of bounded variation such that $\lambda(a) \neq \lambda(b)$, and let $\bar{g} \in[m, M]$. If (6) holds, for all $s \in[\alpha, \beta]$, then

$$
\begin{aligned}
& \frac{\int_{a}^{b} \varphi(g(x)) d \lambda(x)}{\int_{a}^{b} d \lambda(x)}-\varphi(\bar{g}) \\
& \quad \leq \frac{(M-\bar{g})(\bar{g}-m)}{M-m}\left(\varphi_{-}^{\prime}(M)-\varphi_{+}^{\prime}(m)\right) \\
& \quad \leq \frac{1}{4}(M-m)\left(\varphi_{-}^{\prime}(M)-\varphi_{+}^{\prime}(m)\right)
\end{aligned}
$$

holds for every continuous convex function $\varphi:[\alpha, \beta] \rightarrow \mathbb{R}$.

Proof. Let (6) hold for all $s \in[\alpha, \beta]$. Then from Theorem 3 it follows that for every continuous convex function $\varphi$ : $[\alpha, \beta] \rightarrow \mathbb{R}$ we have

$$
\begin{aligned}
& \frac{\int_{a}^{b} \varphi(g(x)) d \lambda(x)}{\int_{a}^{b} d \lambda(x)}-\varphi(\bar{g}) \\
& \quad \leq \frac{M-\bar{g}}{M-m} \varphi(m)+\frac{\bar{g}-m}{M-m} \varphi(M)-\varphi(\bar{g}) .
\end{aligned}
$$

By the convexity of $\varphi$ (as in [4]), we have the gradient inequality

$$
\varphi(t)-\varphi(M) \geq \varphi_{-}^{\prime}(M)(t-M)
$$

for any $t \in[m, M]$. If we multiply this inequality with $t-m \geq$ 0 , we get

$$
\begin{array}{r}
(t-m) \varphi(t)-(t-m) \varphi(M) \geq \varphi_{-}^{\prime}(M)(t-M)(t-m), \\
t \in[m, M] .
\end{array}
$$

Analogously, we get

$$
\begin{array}{r}
(M-t) \varphi(t)-(M-t) \varphi(m) \geq \varphi_{+}^{\prime}(m)(t-m)(M-t), \\
t \in[m, M] .
\end{array}
$$


Adding (12) to (13) and dividing by $M-m$, we get that

$$
\begin{aligned}
& \frac{(t-m) \varphi(M)+(M-t) \varphi(m)}{M-m}-\varphi(t) \\
& \leq \frac{(M-t)(t-m)}{M-m}\left(\varphi_{-}^{\prime}(M)-\varphi_{+}^{\prime}(m)\right)
\end{aligned}
$$

holds for any $t \in[m, M]$. Substituting $t$ with $\bar{g} \in[m, M]$, we obtain the first inequality in (9).

To prove the second inequality in (9), it is enough to notice that the function $h(t)=(M-t)(t-m) /(M-m)$ is concave on $[m, M]$, so for every $t \in[m, M]$ inequality $h(t) \leq(1 / 4)(M-m)$ is valid. That completes the proof of (9). result.

If we set that $m=\alpha$ and $M=\beta$, we get the following

Corollary 6. Let $g:[a, b] \rightarrow \mathbb{R}$ be continuous function and $[\alpha, \beta]$ (where $\alpha \neq \beta$ ) an interval such that the image of $g$ is a subset of $[\alpha, \beta]$. Let $\lambda:[a, b] \rightarrow \mathbb{R}$ be a continuous function or a function of bounded variation such that $\lambda(a) \neq \lambda(b)$, and let $\bar{g} \in[\alpha, \beta]$. If (7) holds for all $s \in[\alpha, \beta]$, then for every continuous convex function $\varphi:[\alpha, \beta] \rightarrow \mathbb{R}$,

$$
\begin{aligned}
& \frac{\int_{a}^{b} \varphi(g(x)) d \lambda(x)}{\int_{a}^{b} d \lambda(x)}-\varphi(\bar{g}) \\
& \quad \leq \frac{(\beta-\bar{g})(\bar{g}-\alpha)}{\beta-\alpha}\left(\varphi_{-}^{\prime}(\beta)-\varphi_{+}^{\prime}(\alpha)\right) \\
& \quad \leq \frac{1}{4}(\beta-\alpha)\left(\varphi_{-}^{\prime}(\beta)-\varphi_{+}^{\prime}(\alpha)\right),
\end{aligned}
$$

provided that $\varphi_{+}^{\prime}(\alpha)$ and $\varphi_{-}^{\prime}(\beta)$ are finite.

Theorem 7. Let $g:[a, b] \rightarrow \mathbb{R}$ be a continuous function and $[\alpha, \beta]$ an interval. Let $m, M \in\langle\alpha, \beta\rangle(m \neq M)$ be such that $m \leq g(t) \leq M$ for all $t \in[a, b]$. Let $\lambda:[a, b] \rightarrow \mathbb{R}$ be a continuous function or a function of bounded variation such that $\lambda(a) \neq \lambda(b)$, and let $\bar{g} \in[m, M]$. If (6) holds for all $s \in[\alpha, \beta]$, then for every continuous convex function $\varphi$ : $[\alpha, \beta] \rightarrow \mathbb{R}$ we have

$$
\begin{aligned}
& \frac{\int_{a}^{b} \varphi(g(x)) d \lambda(x)}{\int_{a}^{b} d \lambda(x)}-\varphi(\bar{g}) \\
& \quad \leq \frac{(M-\bar{g})(\bar{g}-m)}{M-m} \cdot \sup _{t \in(m, M)} \Psi_{\varphi}(t ; m, M) \\
& \quad \leq \frac{(M-\bar{g})(\bar{g}-m)}{M-m}\left(\varphi_{-}^{\prime}(M)-\varphi_{+}^{\prime}(m)\right) \\
& \quad \leq \frac{1}{4}(M-m)\left(\varphi_{-}^{\prime}(M)-\varphi_{+}^{\prime}(m)\right),
\end{aligned}
$$

where $\Psi_{\varphi}(\cdot ; m, M):\langle m, M\rangle \rightarrow \mathbb{R}$ is defined by

$$
\Psi_{\varphi}(t ; m, M)=\frac{\varphi(M)-\varphi(t)}{M-t}-\frac{\varphi(t)-\varphi(m)}{t-m} .
$$

If $\bar{g} \in\langle m, M\rangle$, then we also have

$$
\begin{aligned}
\frac{\int_{a}^{b} \varphi(g(x)) d \lambda(x)}{\int_{a}^{b} d \lambda(x)}-\varphi(\bar{g}) \\
\quad \leq \frac{1}{4}(M-m) \Psi_{\varphi}(\bar{g} ; m, M) \\
\quad \leq \frac{1}{4}(M-m)\left(\varphi_{-}^{\prime}(M)-\varphi_{+}^{\prime}(m)\right) .
\end{aligned}
$$

Proof. Let (6) hold for all $s \in[\alpha, \beta]$. Then from Theorem 3 it follows that

$$
\begin{aligned}
& \frac{\int_{a}^{b} \varphi(g(x)) d \lambda(x)}{\int_{a}^{b} d \lambda(x)}-\varphi(\bar{g}) \\
& \quad \leq \frac{M-\bar{g}}{M-m} \varphi(m)+\frac{\bar{g}-m}{M-m} \varphi(M)-\varphi(\bar{g})
\end{aligned}
$$

holds for every continuous convex function $\varphi:[\alpha, \beta] \rightarrow \mathbb{R}$. If $\bar{g} \in\langle m, M\rangle$, it is easy to verify that the term on the right side of (19) is equal to

$$
\frac{(M-\bar{g})(\bar{g}-m)}{M-m}\left[\frac{\varphi(M)-\varphi(\bar{g})}{M-\bar{g}}-\frac{\varphi(\bar{g})-\varphi(m)}{\bar{g}-m}\right],
$$

and the term in the square brackets in (20) is equal to $\Psi_{\varphi}(\bar{g} ; m, M)$, where the function $\Psi_{\varphi}(\cdot ; m, M)$ is defined in (24). As in [5], it follows that

$$
\begin{aligned}
\Psi_{\varphi}(\bar{g} ; m, M) \leq & \sup _{t \in(m, M)} \Psi_{\varphi}(t ; m, M) \\
= & \sup _{t \in(m, M)}\left[\frac{\varphi(M)-\varphi(t)}{M-t}-\frac{\varphi(t)-\varphi(m)}{t-m}\right] \\
\leq & \sup _{t \in(m, M)}\left[\frac{\varphi(M)-\varphi(t)}{M-t}\right] \\
& +\sup _{t \in(m, M)}\left[-\frac{\varphi(t)-\varphi(m)}{t-m}\right] \\
= & \sup _{t \in(m, M)}\left[\frac{\varphi(M)-\varphi(t)}{M-t}\right] \\
& -\inf _{t \in(m, M)}\left[\frac{\varphi(t)-\varphi(m)}{t-m}\right] \\
= & \varphi_{-}^{\prime}(M)-\varphi_{+}^{\prime}(m),
\end{aligned}
$$

and since

$$
\frac{(M-\bar{g})(\bar{g}-m)}{M-m} \leq \frac{1}{4}(M-m),
$$

the inequality (16) follows. For $\bar{g}=m$ or $\bar{g}=M$, the inequality (16) is obvious. The inequality in (18) follows directly from the proof of inequality (16). 

result.

If we set that $m=\alpha$ and $M=\beta$, we get the following

Corollary 8. Let $g:[a, b] \rightarrow \mathbb{R}$ be a continuous function and $[\alpha, \beta]($ where $\alpha \neq \beta)$ an interval such that the image of $g$ is a subset of $[\alpha, \beta]$. Let $\lambda:[a, b] \rightarrow \mathbb{R}$ be a continuous function or a function of bounded variation such that $\lambda(a) \neq \lambda(b)$, and let $\bar{g} \in[\alpha, \beta]$. If (7) holds for all $s \in[\alpha, \beta]$, then

$$
\begin{aligned}
& \frac{\int_{a}^{b} \varphi(g(x)) d \lambda(x)}{\int_{a}^{b} d \lambda(x)}-\varphi(\bar{g}) \\
& \quad \leq \frac{(\beta-\bar{g})(\bar{g}-\alpha)}{\beta-\alpha} \cdot \sup _{t \in(\alpha, \beta)} \Psi_{\varphi}(t ; \alpha, \beta) \\
& \quad \leq \frac{(\beta-\bar{g})(\bar{g}-\alpha)}{\beta-\alpha}\left(\varphi_{-}^{\prime}(\beta)-\varphi_{+}^{\prime}(\alpha)\right) \\
& \quad \leq \frac{1}{4}(\beta-\alpha)\left(\varphi_{-}^{\prime}(\beta)-\varphi_{+}^{\prime}(\alpha)\right),
\end{aligned}
$$

where $\Psi_{\varphi}(\cdot ; \alpha, \beta):\langle\alpha, \beta\rangle \rightarrow \mathbb{R}$ is defined by

$$
\Psi_{\varphi}(t ; \alpha, \beta)=\frac{\varphi(\beta)-\varphi(t)}{\beta-t}-\frac{\varphi(t)-\varphi(\alpha)}{t-\alpha},
$$

holds for every continuous convex function $\varphi:[\alpha, \beta] \rightarrow \mathbb{R}$, provided that $\varphi_{+}^{\prime}(\alpha)$ and $\varphi_{-}^{\prime}(\beta)$ are finite. If $\bar{g} \in\langle\alpha, \beta\rangle$, then we also have

$$
\begin{aligned}
& \frac{\int_{a}^{b} \varphi(g(x)) d \lambda(x)}{\int_{a}^{b} d \lambda(x)}-\varphi(\bar{g}) \\
& \quad \leq \frac{1}{4}(\beta-\alpha) \Psi_{\varphi}(\bar{g} ; \alpha, \beta) \\
& \quad \leq \frac{1}{4}(\beta-\alpha)\left(\varphi_{-}^{\prime}(\beta)-\varphi_{+}^{\prime}(\alpha)\right) .
\end{aligned}
$$

Theorem 9. Let $g:[a, b] \rightarrow \mathbb{R}$ be a continuous function and $[\alpha, \beta]$ an interval. Let $m, M \in\langle\alpha, \beta\rangle(m \neq M)$ be such that $m \leq g(t) \leq M$ for all $t \in[a, b]$. Let $\lambda:[a, b] \rightarrow \mathbb{R}$ be a continuous function or a function of bounded variation such that $\lambda(a) \neq \lambda(b)$, and let $\bar{g} \in[m, M]$. If (6) holds for all $s \in[\alpha, \beta]$, then for every continuous convex function $\varphi$ : $[\alpha, \beta] \rightarrow \mathbb{R}$ we have

$$
\begin{aligned}
& \frac{\int_{a}^{b} \varphi(g(x)) d \lambda(x)}{\int_{a}^{b} d \lambda(x)}-\varphi(\bar{g}) \\
& \quad \leq \max \left\{\frac{M-\bar{g}}{M-m}, \frac{\bar{g}-m}{M-m}\right\}
\end{aligned}
$$

$$
\begin{aligned}
& \cdot\left[\varphi(m)+\varphi(M)-2 \varphi\left(\frac{m+M}{2}\right)\right] \\
= & \left\{\frac{1}{2}+\frac{1}{M-m}\left|\frac{m+M}{2}-\bar{g}\right|\right\} \\
& \cdot\left[\varphi(m)+\varphi(M)-2 \varphi\left(\frac{m+M}{2}\right)\right] \\
\leq & \frac{1}{2} \max \{M-\bar{g}, \bar{g}-m\}\left\{\varphi_{-}^{\prime}(M)-\varphi_{+}^{\prime}(m)\right\} .
\end{aligned}
$$

In order to prove the theorem, we need the following lemma, which is a special case of $[6$, page 717 , Theorem 1$]$ for $n=2$.

Lemma 10. Let $\varphi$ be a convex function on $D_{\varphi}, x, y \in D_{\varphi}$ and $p, q \in[0,1]$ such that $p+q=1$. Then

$$
\begin{aligned}
\min & \{p, q\}\left[\varphi(x)+\varphi(y)-2 \varphi\left(\frac{x+y}{2}\right)\right] \\
& \leq p \varphi(x)+q \varphi(y)-\varphi(p x+q y) \\
& \leq \max \{p, q\}\left[\varphi(x)+\varphi(y)-2 \varphi\left(\frac{x+y}{2}\right)\right] .
\end{aligned}
$$

Proof of Theorem 9. Let (6) hold for all $s \in[\alpha, \beta]$. Then from Theorem 3 it follows that

$$
\frac{\int_{a}^{b} \varphi(g(x)) d \lambda(x)}{\int_{a}^{b} d \lambda(x)} \leq \frac{M-\bar{g}}{M-m} \varphi(m)+\frac{\bar{g}-m}{M-m} \varphi(M)
$$

holds for every continuous convex function $\varphi:[\alpha, \beta] \rightarrow \mathbb{R}$. Denote $p=(M-\bar{g}) /(M-m)$; so we have $p \in[0,1]$ and $\bar{g}=p \cdot m+(1-p) M$. As in [7], by Lemma 10 we get the following:

$$
\begin{aligned}
\frac{\int_{a}^{b} \varphi(g(x)) d \lambda(x)}{\int_{a}^{b} d \lambda(x)} & -\varphi(\bar{g}) \leq \frac{M-\bar{g}}{M-m} \varphi(m)+\frac{\bar{g}-m}{M-m} \varphi(M)-\varphi(\bar{g}) \\
= & p \varphi(m)+(1-p) \varphi(M)-\varphi(p \cdot m+(1-p) M) \\
\leq & \max \{p, 1-p\} \cdot\left[\varphi(m)+\varphi(M)-2 \varphi\left(\frac{m+M}{2}\right)\right] \\
= & \max \left\{\frac{M-\bar{g}}{M-m}, \frac{\bar{g}-m}{M-m}\right\} \\
& \cdot\left[\varphi(m)+\varphi(M)-2 \varphi\left(\frac{m+M}{2}\right)\right] \\
= & \left\{\frac{1}{2}+\frac{1}{M-m}\left|\frac{m+M}{2}-\bar{g}\right|\right\} \\
& \cdot\left[\varphi(m)+\varphi(M)-2 \varphi\left(\frac{m+M}{2}\right)\right] .
\end{aligned}
$$



that

In order to prove the second inequality, it is easy to verify

$$
\begin{aligned}
& \frac{\varphi(m)+\varphi(M)-2 \varphi((m+M) / 2)}{M-m} \\
& =\frac{1}{2}\left[\frac{\varphi(M)-\varphi((m+M) / 2)}{M-((m+M) / 2)}-\frac{\varphi((m+M) / 2)-\varphi(m)}{((m+M) / 2)-m}\right] .
\end{aligned}
$$

Since $\varphi$ is a convex function on $[\alpha, \beta]$, by the gradient inequality we have (as in [5])

$$
\begin{gathered}
\frac{\varphi(M)-\varphi((m+M) / 2)}{M-((m+M) / 2)} \leq \varphi_{-}^{\prime}(M), \\
\frac{\varphi((m+M) / 2)-\varphi(m)}{((m+M) / 2)-m} \geq \varphi_{+}^{\prime}(m),
\end{gathered}
$$

and we finally obtain

$$
\frac{\varphi(m)+\varphi(M)-2 \varphi((m+M) / 2)}{M-m} \leq \frac{1}{2}\left[\varphi_{-}^{\prime}(M)-\varphi_{+}^{\prime}(m)\right],
$$

so the second inequality in (26) follows. result.

If we set that $m=\alpha$ and $M=\beta$, we get the following

Corollary 11. Let $g:[a, b] \rightarrow \mathbb{R}$ be a continuous function and $[\alpha, \beta]($ where $\alpha \neq \beta)$ an interval such that the image of $g$ is a subset of $[\alpha, \beta]$. Let $\lambda:[a, b] \rightarrow \mathbb{R}$ be a continuous function or a function of bounded variation such that $\lambda(a) \neq \lambda(b)$, and let $\bar{g} \in[\alpha, \beta]$. If (7) holds for all $s \in[\alpha, \beta]$, then for every continuous convex function $\varphi:[\alpha, \beta] \rightarrow \mathbb{R}$ we have

$$
\begin{aligned}
\frac{\int_{a}^{b} \varphi(g(x)) d \lambda(x)}{\int_{a}^{b} d \lambda(x)}-\varphi(\bar{g}) \\
\leq \max \left\{\frac{\beta-\bar{g}}{\beta-\alpha}, \frac{\bar{g}-\alpha}{\beta-\alpha}\right\} \\
\cdot\left[\varphi(\alpha)+\varphi(\beta)-2 \varphi\left(\frac{\alpha+\beta}{2}\right)\right] \\
=\left\{\frac{1}{2}+\frac{1}{\beta-\alpha}\left|\frac{\alpha+\beta}{2}-\bar{g}\right|\right\} \\
\cdot\left[\varphi(\alpha)+\varphi(\beta)-2 \varphi\left(\frac{\alpha+\beta}{2}\right)\right] \\
\leq \frac{1}{2} \max \{\beta-\bar{g}, \bar{g}-\alpha\}\left\{\varphi_{-}^{\prime}(\beta)-\varphi_{+}^{\prime}(\alpha)\right\}
\end{aligned}
$$

provided that $\varphi_{+}^{\prime}(\alpha)$ and $\varphi_{-}^{\prime}(\beta)$ are finite.

Remark 12. If in Theorem 5, Corollary 6, Theorem 7, Corollary 8 , Theorem 9 , and Corollary 11 we also request that
(3) holds for all $s \in[\alpha, \beta]$, then by applying Theorem 1 we will get that

$$
0 \leq \frac{\int_{a}^{b} \varphi(g(x)) d \lambda(x)}{\int_{a}^{b} d \lambda(x)}-\varphi(\bar{g})
$$

also holds for every continuous convex function $\varphi:[\alpha, \beta] \rightarrow$ $\mathbb{R}$.

Remark 13. Result given in Theorem 5 together with Remark 12 represents a generalization of [4, Theorem 2], result given in Theorem 7 together with Remark 12 represents a generalization of [5, Theorem 2], and result given in Theorem 9 with Remark 12 represents a generalization of [5, Theorem 3].

\section{Mean-Value Theorems}

As in the previous section, let $g:[a, b] \rightarrow \mathbb{R}$ be a continuous function and $[\alpha, \beta]$ an interval. Let $m, M \in\langle\alpha, \beta\rangle(m \neq$ $M$ ) be such that $m \leq g(t) \leq M$ for all $t \in[a, b]$. Let $\lambda:[a, b] \rightarrow \mathbb{R}$ be a continuous function or a function of bounded variation such that $\lambda(a) \neq \lambda(b)$, and let $\bar{g} \in[m, M]$.

Motivated by the inequalities (9) and (26) for continuous convex function $\varphi:[\alpha, \beta] \rightarrow \mathbb{R}$, we define functionals $\Phi_{1}(g, \lambda, \varphi)$ and $\Phi_{2}(g, \lambda, \varphi)$ by

$$
\begin{aligned}
\Phi_{1}(g, \lambda, \varphi)= & \frac{(M-\bar{g})(\bar{g}-m)}{M-m}\left(\varphi_{-}^{\prime}(M)-\varphi_{+}^{\prime}(m)\right) \\
& -\frac{\int_{a}^{b} \varphi(g(x)) d \lambda(x)}{\int_{a}^{b} d \lambda(x)}+\varphi(\bar{g}), \\
\Phi_{2}(g, \lambda, \varphi)= & \left\{\frac{1}{2}+\frac{1}{M-m}\left|\frac{m+M}{2}-\bar{g}\right|\right\} \\
& \cdot\left[\varphi(m)+\varphi(M)-2 \varphi\left(\frac{m+M}{2}\right)\right] \\
& -\frac{\int_{a}^{b} \varphi(g(x)) d \lambda(x)}{\int_{a}^{b} d \lambda(x)}+\varphi(\bar{g}) .
\end{aligned}
$$

If (6) holds for all $s \in[\alpha, \beta]$, then from Theorems 5 and 9 it follows that $\Phi_{i}(g, \lambda, \varphi) \geq 0(i=1,2)$. If the function $\varphi$ is concave, that is, if $(6)$ holds with reversed inequality sign for all $s \in[\alpha, \beta]$, then we have $\Phi_{i}(g, \lambda, \varphi) \leq 0(i=1,2)$.

Now we give two mean-value theorems for the functionals $\Phi_{i}(g, \lambda, \varphi)(i=1,2)$. In the following, $\varphi_{0}$ will denote the function defined by $\varphi_{0}(t)=t^{2}$ on whatever domain we need.

Theorem 14. Let $g:[a, b] \rightarrow \mathbb{R}$ be a continuous function and $[\alpha, \beta]$ an interval. Let $m, M \in\langle\alpha, \beta\rangle(m \neq M)$ be such that $m \leq g(t) \leq M$ for all $t \in[a, b]$. Let $\varphi:[\alpha, \beta] \rightarrow \mathbb{R}$, $\varphi \in C^{2}([\alpha, \beta])$. Let $\lambda:[a, b] \rightarrow \mathbb{R}$ be a continuous function or a function of bounded variation such that $\lambda(a) \neq \lambda(b)$ and $\bar{g} \in[m, M]$, and let $\Phi_{1}$ and $\Phi_{2}$ be functionals defined in (35) 
and (36), respectively. Then there exist $\xi_{1}, \xi_{2} \in[\alpha, \beta]$ such that the following equalities hold:

$$
\Phi_{i}(g, \lambda, \varphi)=\frac{\varphi^{\prime \prime}\left(\xi_{i}\right)}{2} \Phi_{i}\left(g, \lambda, \varphi_{0}\right), \quad i=1,2 .
$$

Proof. We only give the proof for the functional $\Phi_{1}$, since both proofs have the same concept. Since $\varphi \in C^{2}([\alpha, \beta])$, there exist $m_{1}=\min _{t \in[\alpha, \beta]} \varphi^{\prime \prime}(t)$ and $M_{1}=\max _{t \in[\alpha, \beta]} \varphi^{\prime \prime}(t)$. It is easy to verify that the functions $\varphi_{1}, \varphi_{2}$ defined by

$$
\begin{aligned}
& \varphi_{1}(t)=\frac{M_{1}}{2} t^{2}-\varphi(t), \\
& \varphi_{2}(t)=\varphi(t)-\frac{m_{1}}{2} t^{2}
\end{aligned}
$$

are continuous and convex, and therefore $\Phi_{1}\left(g, \lambda, \varphi_{1}\right) \geq 0$, $\Phi_{1}\left(g, \lambda, \varphi_{2}\right) \geq 0$. This implies that

$$
\frac{m_{1}}{2} \Phi_{1}\left(g, \lambda, \varphi_{0}\right) \leq \Phi_{1}(g, \lambda, \varphi) \leq \frac{M_{1}}{2} \Phi_{1}\left(g, \lambda, \varphi_{0}\right) .
$$

Hence, as the function $\varphi^{\prime \prime}$ is continuous, there exists $\xi_{1} \in$ $[\alpha, \beta]$ such that

$$
\Phi_{1}(g, \lambda, \varphi)=\frac{\varphi^{\prime \prime}\left(\xi_{1}\right)}{2} \Phi_{1}\left(g, \lambda, \varphi_{0}\right) .
$$

The proof for the functional $\Phi_{2}$ is analogous.

Theorem 15. Let $g:[a, b] \rightarrow \mathbb{R}$ be a continuous function and $[\alpha, \beta]$ an interval. Let $m, M \in\langle\alpha, \beta\rangle(m \neq M)$ be such that $m \leq g(t) \leq M$ for all $t \in[a, b]$. Let $\varphi, \psi:[\alpha, \beta] \rightarrow \mathbb{R}$, $\varphi, \psi \in C^{2}([\alpha, \beta])$. Let $\lambda:[a, b] \rightarrow \mathbb{R}$ be a continuous function or a function of bounded variation such that $\lambda(a) \neq \lambda(b)$ and $\bar{g} \in[m, M]$, and let $\Phi_{1}$ and $\Phi_{2}$ be functionals defined in (35) and (36), respectively. Then there exist $\xi_{1}, \xi_{2} \in[\alpha, \beta]$ such that the following equalities hold

$$
\frac{\Phi_{i}(g, \lambda, \varphi)}{\Phi_{i}(g, \lambda, \psi)}=\frac{\varphi^{\prime \prime}\left(\xi_{i}\right)}{\psi^{\prime \prime}\left(\xi_{i}\right)}, \quad i=1,2,
$$

provided that the denominators are nonzero.

Proof. We give the proof for the functional $\Phi_{1}$. Define the function $\chi$ as a linear combination of functions $\varphi$ and $\psi$

$$
\begin{aligned}
\chi=c_{1} \cdot \varphi-c_{2} \cdot \psi, \quad \text { where } c_{1} & =\Phi_{1}(g, \lambda, \psi), \\
c_{2} & =\Phi_{1}(g, \lambda, \varphi) .
\end{aligned}
$$

Now, applying previous theorem on our function $\chi$, we obtain that there exists $\xi_{1} \in[\alpha, \beta]$ such that

$$
\left(c_{1} \frac{\varphi^{\prime \prime}\left(\xi_{1}\right)}{2}-c_{2} \frac{\psi^{\prime \prime}\left(\xi_{1}\right)}{2}\right) \Phi_{1}\left(g, \lambda, \varphi_{0}\right)=0 .
$$

Since $\Phi_{1}\left(g, \lambda, \varphi_{0}\right) \neq 0$ (otherwise we would have a contradiction with $\left.\Phi_{1}(g, \lambda, \psi) \neq 0\right)$, we get

$$
\frac{\Phi_{1}(g, \lambda, \varphi)}{\Phi_{1}(g, \lambda, \psi)}=\frac{\varphi^{\prime \prime}\left(\xi_{1}\right)}{\psi^{\prime \prime}\left(\xi_{1}\right)} .
$$

The proof for the functional $\Phi_{2}$ is analogous.
Remark 16. If the inverse of the function $\varphi^{\prime \prime} / \psi^{\prime \prime}$ exists, then (41) gives

$$
\xi_{i}=\left(\frac{\varphi^{\prime \prime}}{\psi^{\prime \prime}}\right)^{-1}\left(\frac{\Phi_{i}(g, \lambda, \varphi)}{\Phi_{i}(g, \lambda, \psi)}\right) \in[\alpha, \beta], \quad i=1,2 .
$$

Remark 17. Theorems 14 and 15 also hold if we set $m=\alpha$ and $M=\beta$; that is, if we extend the image of the function $g$ to the entire interval $[\alpha, \beta]$, provided that $\varphi_{+}^{\prime}(\alpha), \varphi_{-}^{\prime}(\beta), \psi_{+}^{\prime}(\alpha)$, and $\psi_{+}^{\prime}(\alpha)$ are finite.

\section{4. $n$-Exponential Convexity}

At the beginning of this section, let us recall some definitions and facts about exponentially convex functions (for instance, see [8] or [9]).

Definition 18. A function $f: I \rightarrow \mathbb{R}$ is $n$-exponentially convex in the Jensen sense on $I$ if

$$
\sum_{i, j=1}^{n} p_{i} p_{j} f\left(\frac{x_{i}+x_{j}}{2}\right) \geq 0
$$

holds for all $p_{i} \in \mathbb{R}$ and $x_{i} \in I, i=1, \ldots, n$.

A function $f: I \rightarrow \mathbb{R}$ is $n$-exponentially convex if it is $n$ exponentially convex in the Jensen sense and continuous on I.

Remark 19. We can see from the definition that 1exponentially convex functions in the Jensen sense are in fact nonnegative functions. Also, $n$-exponentially convex functions in the Jensen sense are $k$-exponentially convex in the Jensen sense for every $k \in \mathbb{N}, k \leq n$.

By definition of the positive semidefinite matrices and some basic linear algebra, we have the following result.

Lemma 20. If $f$ is an n-exponentially convex function in the Jensen sense, then the matrix $\left[f\left(\left(x_{i}+x_{j}\right) / 2\right)\right]_{i, j=1}^{k}$ is positive semidefinite for all $k \in \mathbb{N}, k \leq n$. Particularly, $\operatorname{det}\left[f\left(\left(x_{i}+x_{j}\right) / 2\right)\right]_{i, j=1}^{k} \geq 0$ for all $k \in \mathbb{N}, k \leq n$.

Definition 21. A function $f: I \rightarrow \mathbb{R}$ is exponentially convex in the Jensen sense on $I$, if it is $n$-exponentially convex in the Jensen sense for all $n \in \mathbb{N}$.

A function $f: I \rightarrow \mathbb{R}$ is exponentially convex if it is exponentially convex in the Jensen sense and continuous.

Remark 22. Some examples of exponentially convex functions are as follows (see [10]):

(i) $f: I \rightarrow \mathbb{R}$ defined by $f(x)=c e^{k x}$, where $c \geq 0$ and $k \in \mathbb{R}$,

(ii) $f: \mathbb{R}^{+} \rightarrow \mathbb{R}$ defined by $f(x)=x^{-k}$, where $k>0$,

(iii) $f: \mathbb{R}^{+} \rightarrow \mathbb{R}^{+}$defined by $f(x)=e^{-k \sqrt{x}}$, where $k>0$. 
Remark 23. It is known that a function $f: I \rightarrow \mathbb{R}^{+}$is logconvex in the Jensen sense on $I$ if and only if the relation

$$
\alpha^{2} f(x)+2 \alpha \beta f\left(\frac{x+y}{2}\right)+\beta^{2} f(y) \geq 0
$$

holds for every $\alpha, \beta \in \mathbb{R}$ and $x, y \in I$. It follows that a positive function is log-convex in the Jensen sense if and only if it is 2 -exponentially convex in the Jensen sense. Also, using basic theory of convex functions, it follows that a positive function is log-convex if and only if it is 2-exponentially convex.

The following lemma is equivalent to the definition of convex function (see [3], page 2).

Lemma 24. If $x_{1}, x_{2}, x_{3} \in I$ are such that $x_{1}<x_{2}<x_{3}$, then the function $f: I \rightarrow \mathbb{R}$ is convex if and only if the following inequality holds:

$$
\left(x_{3}-x_{2}\right) f\left(x_{1}\right)+\left(x_{1}-x_{3}\right) f\left(x_{2}\right)+\left(x_{2}-x_{1}\right) f\left(x_{3}\right) \geq 0 \text {. }
$$

We will also need the following result (see [3], page 2).

Lemma 25. If $f: I \rightarrow \mathbb{R}$ is a convex function and $x_{1}, x_{2}, y_{1}, y_{2} \in I$ are such that $x_{1} \leq y_{1}, x_{2} \leq y_{2}, x_{1} \neq x_{2}$, and $y_{1} \neq y_{2}$, then the following inequality is valid:

$$
\frac{f\left(x_{2}\right)-f\left(x_{1}\right)}{x_{2}-x_{1}} \leq \frac{f\left(y_{2}\right)-f\left(y_{1}\right)}{y_{2}-y_{1}} .
$$

If the function $f$ is concave, then the reverse inequality in (49) holds.

When dealing with functions with different degrees of smoothness, divided differences are found to be very useful.

Definition 26. The second order divided difference of a function $f: I \rightarrow \mathbb{R}$ at mutually different points $y_{0}, y_{1}, y_{2} \in I$ is defined recursively by

$$
\begin{aligned}
{\left[y_{i}\right] f } & =f\left(y_{i}\right), \quad i=0,1,2, \\
{\left[y_{i}, y_{i+1}\right] f } & =\frac{f\left(y_{i+1}\right)-f\left(y_{i}\right)}{y_{i+1}-y_{i}}, \quad i=0,1, \\
{\left[y_{0}, y_{1}, y_{2}\right] f } & =\frac{\left[y_{1}, y_{2}\right] f-\left[y_{0}, y_{1}\right] f}{y_{2}-y_{0}} .
\end{aligned}
$$

Remark 27. The value $\left[y_{0}, y_{1}, y_{2}\right] f$ is independent of the order of the points $y_{0}, y_{1}$, and $y_{2}$. This definition may be extended to include the case in which some or all of the points coincide (see [3], page 16). Taking the limit $y_{1} \rightarrow y_{0}$ in (50), we get

$$
\begin{aligned}
\lim _{y_{1} \rightarrow y_{0}} & {\left[y_{0}, y_{1}, y_{2}\right] f } \\
& =\left[y_{0}, y_{0}, y_{2}\right] f \\
& =\frac{f\left(y_{2}\right)-f\left(y_{0}\right)-f^{\prime}\left(y_{0}\right)\left(y_{2}-y_{0}\right)}{\left(y_{2}-y_{0}\right)^{2}}, \quad y_{2} \neq y_{0},
\end{aligned}
$$

provided that $f^{\prime}$ exists. Furthermore, taking the limits $y_{i} \rightarrow$ $y_{0}, i=1,2$ in (50), we get

$$
\lim _{y_{2} \rightarrow y_{0}} \lim _{y_{1} \rightarrow y_{0}}\left[y_{0}, y_{1}, y_{2}\right] f=\left[y_{0}, y_{0}, y_{0}\right] f=\frac{f^{\prime \prime}\left(y_{0}\right)}{2},
$$

provided that $f^{\prime \prime}$ exists.

A function $f: I \rightarrow \mathbb{R}$ is convex if and only if for every choice of three mutually different points $y_{0}, y_{1}, y_{2} \in$ $I\left[y_{0}, y_{1}, y_{2}\right] f \geq 0$ holds.

Now, we use an idea from [10] to give an elegant method of producing $n$-exponentially convex functions and exponentially convex functions by applying functionals $\Phi_{i}(i=1,2)$ to a given family of functions with the same property.

For the rest of this section we assume that (6) holds, so from Theorems 5 and 9 it follows that $\Phi_{i}(g, \lambda, \varphi) \geq 0(i=$ $1,2)$ under the appropriate assumptions on functions $g, \lambda$, and $\varphi$.

Theorem 28. Let $g:[a, b] \rightarrow \mathbb{R}$ be a continuous function and $[\alpha, \beta]$ an interval. Let $m, M \in\langle\alpha, \beta\rangle(m \neq M)$ be such that $m \leq g(t) \leq M$ for all $t \in[a, b]$. Let $\Omega=\left\{\varphi_{p}: p \in J\right\}$ (where $J$ is an interval in $\mathbb{R}$ ) be a family of functions $\varphi_{p}$ : $[\alpha, \beta] \rightarrow \mathbb{R}, \varphi_{p} \in C([\alpha, \beta])$, such that the function $p \mapsto$ $\left[y_{0}, y_{1}, y_{2}\right] \varphi_{p}$ is n-exponentially convex in the Jensen sense on $J$ for every three mutually different points $y_{0}, y_{1}, y_{2} \in[\alpha, \beta]$. Let $\lambda:[a, b] \rightarrow \mathbb{R}$ be a continuous function or a function of bounded variation such that $\lambda(a) \neq \lambda(b)$, let $\bar{g} \in[m, M]$, and let $\Phi_{1}$ and $\Phi_{2}$ be linear functionals defined in (35) and (36), respectively. Then the function $p \mapsto \Phi_{i}\left(g, \lambda, \varphi_{p}\right)(i=1,2)$ is $n$-exponentially convex in the Jensen sense on J. If the function $p \mapsto \Phi_{i}\left(g, \lambda, \varphi_{p}\right)$ is continuous on $J$, then it is n-exponentially convex on $\mathrm{J}$.

Proof. For $q_{j} \in \mathbb{R}(j=1, \ldots, n)$ we define the function

$$
h(x)=\sum_{j, k=1}^{n} q_{j} q_{k} \varphi_{\left(p_{j}+p_{k}\right) / 2}(x),
$$

where $p_{j}, p_{k} \in J, 1 \leq j, k \leq n$, and $\varphi_{\left(p_{j}+p_{k}\right) / 2} \in \Omega$. Since $p \rightarrow\left[y_{0}, y_{1}, y_{2}\right] \varphi_{p}$ is $n$-exponentially convex in the Jensen sense by assumption, for every three mutually different points $y_{0}, y_{1}, y_{2} \in[\alpha, \beta]$, we have

$$
\left[y_{0}, y_{1}, y_{2}\right] h=\sum_{j, k=1}^{n} q_{j} q_{k}\left[y_{0}, y_{1}, y_{2}\right] \varphi_{\left(p_{j}+p_{k}\right) / 2} \geq 0 \text {. }
$$

It follows that $h$ is convex (and continuous) function on $[\alpha, \beta]$, so

$$
\Phi_{i}(g, \lambda, h) \geq 0
$$

and hence

$$
\sum_{j, k=1}^{n} q_{j} q_{k} \Phi_{i}\left(g, \lambda, \varphi_{\left(p_{j}+p_{k}\right) / 2}\right) \geq 0 .
$$

We conclude that the function $p \mapsto \Phi_{i}\left(g, \lambda, \varphi_{p}\right)$ is $n$ exponentially convex on $J$ in the Jensen sense.

If the function $p \mapsto \Phi_{i}\left(g, \lambda, \varphi_{p}\right)$ is also continuous on $J$, then it is $n$-exponentially convex by definition. 
The following corollary is an immediate consequence of the above theorem.

Corollary 29. Let $g:[a, b] \rightarrow \mathbb{R}$ be a continuous function and $[\alpha, \beta]$ an interval. Let $m, M \in\langle\alpha, \beta\rangle(m \neq M)$ be such that $m \leq g(t) \leq M$ for all $t \in[a, b]$. Let $\Omega=\left\{\varphi_{p}:\right.$ $p \in J\}$ (where $J$ is an interval in $\mathbb{R}$ ) be a family of functions $\varphi_{p}:[\alpha, \beta] \rightarrow \mathbb{R}, \varphi_{p} \in C([\alpha, \beta])$, such that the function $p \mapsto\left[y_{0}, y_{1}, y_{2}\right] \varphi_{p}$ is exponentially convex in the Jensen sense on $J$ for every three mutually different points $y_{0}, y_{1}, y_{2} \in[\alpha, \beta]$. Let $\lambda:[a, b] \rightarrow \mathbb{R}$ be a continuous function or a function of bounded variation such that $\lambda(a) \neq \lambda(b)$, let $\bar{g} \in[m, M]$, and let $\Phi_{1}$ and $\Phi_{2}$ be linear functionals defined in (35) and (36), respectively. Then the function $p \mapsto \Phi_{i}\left(g, \lambda, \varphi_{p}\right)(i=1,2)$ is exponentially convex in the Jensen sense on J. If the function $p \mapsto \Phi_{i}\left(g, \lambda, \varphi_{p}\right)$ is continuous on $J$, then it is exponentially convex on $J$.

Corollary 30. Let $g:[a, b] \rightarrow \mathbb{R}$ be a continuous function and $[\alpha, \beta]$ an interval. Let $m, M \in\langle\alpha, \beta\rangle(m \neq M)$ be such that $m \leq g(t) \leq M$ for all $t \in[a, b]$. Let $\Omega=\left\{\varphi_{p}: p \in J\right\}$ (where $J$ is an interval in $\mathbb{R}$ ) be a family of functions $\varphi_{p}$ : $[\alpha, \beta] \rightarrow \mathbb{R}, \varphi_{p} \in C([\alpha, \beta])$, such that the function $p \mapsto$ $\left[y_{0}, y_{1}, y_{2}\right] \varphi_{p}$ is 2-exponentially convex in the Jensen sense on $J$ for every three mutually different points $y_{0}, y_{1}, y_{2} \in[\alpha, \beta]$. Let $\lambda:[a, b] \rightarrow \mathbb{R}$ be a continuous function or a function of bounded variation such that $\lambda(a) \neq \lambda(b)$, let $\bar{g} \in[m, M]$, and let $\Phi_{1}$ and $\Phi_{2}$ be linear functionals defined in (35) and (36), respectively.

Then the following statements hold.

(i) If the function $p \mapsto \Phi_{i}\left(g, \lambda, \varphi_{p}\right)$ is continuous on $J$, then it is 2-exponentially convex on J. If $p \mapsto$ $\Phi_{i}\left(g, \lambda, \varphi_{p}\right)$ is additionally strictly positive, then it is also log-convex on $J$, and for $r, s, t \in J$ such that $r<$ $s<t$, we have

$\left(\Phi_{i}\left(g, \lambda, \varphi_{s}\right)\right)^{t-r} \leq\left(\Phi_{i}\left(g, \lambda, \varphi_{r}\right)\right)^{t-s}\left(\Phi_{i}\left(g, \lambda, \varphi_{t}\right)\right)^{s-r}$.

(ii) If the function $p \mapsto \Phi_{i}\left(g, \lambda, \varphi_{p}\right)$ is strictly positive and differentiable on $J$, then for every $p, q, u, v \in J$ such that $p \leq u$ and $q \leq v$, we have

$\mu_{p, q}\left(g, \Phi_{i}, \Omega\right) \leq \mu_{u, v}\left(g, \Phi_{i}, \Omega\right), \quad(i=1,2)$,

where

$$
\mu_{p, q}\left(g, \Phi_{i}, \Omega\right)= \begin{cases}\left(\frac{\Phi_{i}\left(g, \lambda, \varphi_{p}\right)}{\Phi_{i}\left(g, \lambda, \varphi_{q}\right)}\right)^{1 /(p-q)}, & p \neq q \\ \exp \left(\frac{(d / d p) \Phi_{i}\left(g, \lambda, \varphi_{p}\right)}{\Phi_{i}\left(g, \lambda, \varphi_{p}\right)}\right), & p=q\end{cases}
$$

$$
\operatorname{for} \varphi_{p}, \varphi_{q} \in \Omega
$$

Proof. (i) The first part is an immediate consequence of Theorem 28, and in the second part, log-convexity is an immediate consequence of Remark 23. Since $p \mapsto$ $\Phi_{i}\left(g, \lambda, \varphi_{p}\right)$ is strictly positive, then by applying Lemma 24 on the function $f(x)=\log \Phi_{i}\left(g, \lambda, \varphi_{x}\right)$ and $r, s, t \in J(r<s<t)$ we get

$$
\begin{gathered}
(t-s) \Phi_{i}\left(g, \lambda, \varphi_{r}\right)+(r-t) \Phi_{i}\left(g, \lambda, \varphi_{s}\right) \\
+(s-r) \Phi_{i}\left(g, \lambda, \varphi_{t}\right) \geq 0,
\end{gathered}
$$

which is equivalent to inequality (57).

(ii) Since by (i) the function $p \mapsto \Phi_{i}\left(g, \lambda, \varphi_{p}\right)$ is logconvex on $J$, that is, the function $p \mapsto \log \Phi_{i}\left(g, \lambda, \varphi_{p}\right)$ is convex on $J$, by applying Lemma 25 for $p \leq u, q \leq v, p \neq q$, and $u \neq v$, we get

$$
\begin{array}{r}
\frac{\log \Phi_{i}\left(g, \lambda, \varphi_{p}\right)-\log \Phi_{i}\left(g, \lambda, \varphi_{q}\right)}{p-q} \\
\leq \frac{\log \Phi_{i}\left(g, \lambda, \varphi_{u}\right)-\log \Phi_{i}\left(g, \lambda, \varphi_{v}\right)}{u-v},
\end{array}
$$

and therefore we conclude that

$$
\mu_{p, q}\left(g, \Phi_{i}, \Omega\right) \leq \mu_{u, v}\left(g, \Phi_{i}, \Omega\right) .
$$

The cases $p=q$ and $u=v$ follow from (61) as limit cases.

Remark 31. Note that the results from Theorem 28 and Corollaries 29 and 30 still hold when two of the points $y_{0}, y_{1}, y_{2} \in[\alpha, \beta]$ coincide (say $y_{1}=y_{0}$ ), for a family of differentiable functions $\varphi_{p}$ such that the function $p \mapsto$ $\left[y_{0}, y_{1}, y_{2}\right] \varphi_{p}$ is $n$-exponentially convex in the Jensen sense (exponentially convex in the Jensen sense, log-convex in the Jensen sense). Furthermore, these results still hold when all three points coincide for a family of twice differentiable functions with the above mentioned properties. The proofs are obtained by recalling Remark 27 and suitable characterization of convexity.

Remark 32. The results from this section also hold if we set $m=\alpha$ and $M=\beta$, that is, if we extend the image of the function $g$ to the entire interval $[\alpha, \beta]$, provided that $\varphi_{+}^{\prime}(\alpha)$ and $\varphi_{-}^{\prime}(\beta)$ are finite.

\section{Examples}

In this section we will vary on choice of a family $\Omega=\left\{\varphi_{p}\right.$ : $p \in J\}$, presenting several families of functions which fulfil the conditions of Theorem 28 and Corollaries 29 and 30 (and Remark 31). This enables us to construct different examples of exponentially convex functions and to construct some Cauchy-type means.

Example 1. Let

$$
\Omega_{1}=\left\{\psi_{p}: \mathbb{R} \rightarrow[0, \infty): p \in \mathbb{R}\right\}
$$

be a family of functions defined by

$$
\psi_{p}(x)= \begin{cases}\frac{1}{p^{2}} e^{p x}, & p \neq 0 ; \\ \frac{1}{2} x^{2}, & p=0 .\end{cases}
$$


Since $\left(d^{2} / d x^{2}\right) \psi_{p}(x)=e^{p x}>0$ for $x \in \mathbb{R}, \psi_{p}$ is convex function on $\mathbb{R}$ for every $p \in \mathbb{R}$. From Remark 22 it follows that the function $p \mapsto\left(d^{2} / d x^{2}\right) \psi_{p}(x)$ is exponentially convex, and from [10] we then also have that $p \mapsto\left[y_{0}, y_{1}, y_{2}\right] \psi_{p}$ is exponentially convex (and so exponentially convex in the Jensen sense). So, our family $\Omega_{1}$ of functions $\psi_{p}$ fulfills the condition given in Corollary 29, and we conclude that $p \mapsto$ $\Phi_{i}\left(g, \lambda, \psi_{p}\right)$ (for $i=1,2$ ) are exponentially convex in the Jensen sense. It is easy to verify that these mappings are continuous (although $p \mapsto \psi_{p}$ is not continuous at $p=0$ ), so they are exponentially convex.

Using Corollary 30 for this family of functions, $\mu_{p, q}\left(g, \Phi_{i}, \Omega_{1}\right)(i=1,2)$ from (59) become

$$
\begin{aligned}
\mu_{p, q}\left(g, \Phi_{i}, \Omega_{1}\right) & \begin{array}{ll}
\left(\frac{\Phi_{i}\left(g, \lambda, \psi_{p}\right)}{\Phi_{i}\left(g, \lambda, \psi_{q}\right)}\right)^{1 /(p-q)}, & p \neq q ; \\
\exp \left(\frac{\Phi_{i}\left(g, \lambda, i d \cdot \psi_{p}\right)}{\Phi_{i}\left(g, \lambda, \psi_{p}\right)}-\frac{2}{p}\right), & p=q \neq 0 ; \\
\exp \left(\frac{1}{3} \frac{\Phi_{i}\left(g, \lambda, i d \cdot \psi_{0}\right)}{\Phi_{i}\left(g, \lambda, \psi_{0}\right)}\right), & p=q=0 ;
\end{array}
\end{aligned}
$$

and using (58) we have that they are monotonous in parameters $p$ and $q$.

If $\Phi_{i}(i=1,2)$ are positive, then using Theorem 15 applied for $\varphi=\psi_{p} \in \Omega_{1}$ and $\psi=\psi_{q} \in \Omega_{1}$, it follows that

$$
M_{p, q}\left(g, \Phi_{i}, \Omega_{1}\right)=\log \mu_{p, q}\left(g, \Phi_{i}, \Omega_{1}\right), \quad \text { for } i=1,2,
$$

satisfy

$$
\alpha \leq M_{p, q}\left(g, \Phi_{i}, \Omega_{1}\right) \leq \beta, \quad \text { for } i=1,2 .
$$

If we set that the image of the function $g$ is $[\alpha, \beta]$, then we have

$$
\begin{aligned}
\alpha & =\min _{t \in[a, b]}\{g(t)\} \leq M_{p, q}\left(g, \Phi_{i}, \Omega_{1}\right) \\
& \leq \max _{t \in[a, b]}\{g(t)\}=\beta, \quad \text { for } i=1,2,
\end{aligned}
$$

which shows that in this case $M_{p, q}\left(g, \Phi_{i}, \Omega_{1}\right)$ are means (of the function $g)$. Notice that by $(58) M_{p, q}\left(g, \Phi_{i}, \Omega_{1}\right)$ are also monotonic.

Example 2. Let

$$
\Omega_{2}=\left\{\varphi_{p}: \mathbb{R}^{+} \longrightarrow \mathbb{R}: p \in \mathbb{R}\right\}
$$

be a family of functions defined by

$$
\varphi_{p}(x)= \begin{cases}\frac{x^{p}}{p(p-1)}, & p \neq 0,1 ; \\ -\log x, & p=0 ; \\ x \log x, & p=1 .\end{cases}
$$

Since $\left(d^{2} / d x^{2}\right) \varphi_{p}(x)=x^{p-2}=e^{(p-2) \log x}>0, \varphi_{p}$ is convex function for $x>0$. From Remark 22 it follows that $p \mapsto\left(d^{2} / d x^{2}\right) \varphi_{p}(x)$ is exponentially convex, and from [10] we then also have that $p \mapsto\left[y_{0}, y_{1}, y_{2}\right] \varphi_{p}$ is exponentially convex (and so exponentially convex in the Jensen sense). So, our family $\Omega_{2}$ of functions $\varphi_{p}$ fulfills the condition given in Corollary 29.

In this example we assume that our interval $[\alpha, \beta]$ from Corollaries 29 and 30 is a subset of $\mathbb{R}^{+}$, and for our family of functions we have the following:

$$
\begin{aligned}
& \mu_{p, q}\left(g, \Phi_{i}, \Omega_{2}\right) \\
& = \begin{cases}\left(\frac{\Phi_{i}\left(g, \lambda, \varphi_{p}\right)}{\Phi_{i}\left(g, \lambda, \varphi_{q}\right)}\right)^{1 /(p-q)}, & p \neq q ; \\
\exp \left(\frac{1-2 p}{p(p-1)}-\frac{\Phi_{i}\left(g, \lambda, \varphi_{0} \varphi_{p}\right)}{\Phi_{i}\left(g, \lambda, \varphi_{p}\right)}\right), & p=q \neq 1,0 ; \\
\exp \left(1-\frac{\Phi_{i}\left(g, \lambda, \varphi_{0}^{2}\right)}{2 \Phi_{i}\left(g, \lambda, \varphi_{0}\right)}\right), & p=q=0 . \\
\exp \left(-1-\frac{\Phi_{i}\left(g, \lambda, \varphi_{0} \varphi_{1}\right)}{2 \Phi_{i}\left(g, \lambda, \varphi_{1}\right)}\right), & p=q=1 .\end{cases}
\end{aligned}
$$

As in the previous example, we conclude that functions $p \mapsto \Phi_{i}\left(g, \lambda, \varphi_{p}\right)(i=1,2)$ are exponentially convex and that for $\mu_{p, q}\left(g, \Phi_{i}, \Omega_{2}\right)$ the monotonicity property holds.

If $\Phi_{i}(i=1,2)$ are positive, then Theorem 15 applied for $\varphi=\varphi_{p} \in \Omega_{2}$ and $\psi=\varphi_{q} \in \Omega_{2}$ yields that there exist

$$
\xi_{i} \in[\alpha, \beta], \quad(i=1,2),
$$

such that

$$
\xi_{i}^{p-q}=\frac{\Phi_{i}\left(g, \lambda, \varphi_{p}\right)}{\Phi_{i}\left(g, \lambda, \varphi_{q}\right)}
$$

Since the function $\xi \mapsto \xi^{p-q}$ is invertible for $p \neq q$, we then have

$$
\alpha \leq\left(\frac{\Phi_{i}\left(g, \lambda, \varphi_{p}\right)}{\Phi_{i}\left(g, \lambda, \varphi_{q}\right)}\right)^{1 /(p-q)} \leq \beta, \quad \text { for } i=1,2 .
$$

As in the previous example, if we set that the image of the function $g$ is $[\alpha, \beta]$, then we have

$$
\begin{aligned}
\alpha & =\min _{t \in[a, b]}\{g(t)\} \leq\left(\frac{\Phi_{i}\left(g, \lambda, \varphi_{p}\right)}{\Phi_{i}\left(g, \lambda, \varphi_{q}\right)}\right)^{1 /(p-q)} \\
& \leq \max _{t \in[a, b]}\{g(t)\}=\beta,
\end{aligned}
$$

which shows that in this case $\mu_{p, q}\left(g, \Phi_{i}, \Omega_{2}\right)$ are means (of function $g$ ). 
Now, we impose one additional parameter $r$. For $r \neq 0$ by substituting $g \rightarrow g^{r}, p \rightarrow p / r$, and $q \rightarrow q / r$ in (75), we get

$$
\begin{aligned}
\min _{t \in[a, b]}\left\{(g(t))^{r}\right\} & \leq\left(\frac{\Phi_{i}\left(g^{r}, \lambda, \varphi_{p}\right)}{\Phi_{i}\left(g^{r}, \lambda, \varphi_{q}\right)}\right)^{r /(p-q)} \\
& \leq \max _{t \in[a, b]}\left\{(g(t))^{r}\right\}, \quad \text { for } i=1,2 .
\end{aligned}
$$

We define new generalized mean as follows:

$$
\mu_{p, q ; r}\left(g, \Phi_{i}, \Omega_{2}\right)= \begin{cases}\left(\mu_{p / r, q / r}\left(g^{r}, \Phi_{i}, \Omega_{2}\right)\right)^{1 / r}, & r \neq 0 ; \\ \mu_{p, q}\left(\log g, \Phi_{i}, \Omega_{1}\right), & r=0 .\end{cases}
$$

These new generalized means are also monotonic. If $p, q, u, v \in \mathbb{R}, r \neq 0$ such that $p \leq u, q \leq v$, then we have

$$
\mu_{p, q ; r}\left(g, \Phi_{i}, \Omega_{2}\right) \leq \mu_{u, v ; r}\left(g, \Phi_{i}, \Omega_{2}\right), \quad \text { for } i=1,2 .
$$

This result follows from

$$
\begin{aligned}
\mu_{p / r, q / r}\left(g^{r}, \Phi_{i}, \Omega_{2}\right) & =\left(\frac{\Phi_{i}\left(g^{r}, \lambda, \varphi_{p / r}\right)}{\Phi_{i}\left(g^{r}, \lambda, \varphi_{q / r}\right)}\right)^{r /(p-q)} \\
& \leq\left(\frac{\Phi_{i}\left(g^{r}, \lambda, \varphi_{u / r}\right)}{\Phi_{i}\left(g^{r}, \lambda, \varphi_{v / r}\right)}\right)^{r /(u-v)} \\
& =\mu_{u / r, v / r}\left(g^{r}, \Phi_{i}, \Omega_{2}\right)
\end{aligned}
$$

for $p, q, u, v \in \mathbb{R}, r \neq 0$, such that $p / r \leq u / r, q / r \leq v / r$, and the fact that $\mu_{p, q}\left(g, \Phi_{i}, \Omega_{2}\right)$ for $i=1,2$ are monotonous in both parameters. For $r=0$, we obtain the required result by taking the limit $r \rightarrow 0$.

Example 3. Let

$$
\Omega_{3}=\left\{\theta_{p}: \mathbb{R}^{+} \longrightarrow \mathbb{R}^{+}: p \in \mathbb{R}^{+}\right\}
$$

be a family of functions defined by

$$
\theta_{p}(x)=\frac{e^{-x \sqrt{p}}}{p} .
$$

Since $\left(d^{2} / d x^{2}\right) \theta_{p}(x)=e^{-x \sqrt{p}}>0, \theta_{p}$ is convex function for $x>0$. From Remark 22 we have that $p \mapsto\left(d^{2} / d x^{2}\right) \theta_{p}(x)$ is exponentially convex, and from [10] we then also have that $p \mapsto\left[y_{0}, y_{1}, y_{2}\right] \theta_{p}$ is exponentially convex function. Family $\Omega_{3}$ of functions $\theta_{p}$ fulfills the condition given in Corollary 29. Here in this example we again assume that interval $[\alpha, \beta]$ from our corollaries is a subset of $\mathbb{R}^{+}$, and so for our family of functions we have the following possible cases for $\mu_{p, q}$ :

$$
\begin{aligned}
\mu_{p, q}\left(g, \Phi_{i}, \Omega_{3}\right) & \begin{cases}\left(\frac{\Phi_{i}\left(g, \lambda, \theta_{p}\right)}{\Phi_{i}\left(g, \lambda, \theta_{q}\right)}\right)^{1 /(p-q)}, & p \neq q ; \\
\exp \left(-\frac{\Phi_{i}\left(g, \lambda, i d \cdot \theta_{p}\right)}{2 \sqrt{p} \Phi_{i}\left(g, \lambda, \theta_{p}\right)}-\frac{1}{p}\right), & p=q .\end{cases}
\end{aligned}
$$

As before, we conclude that the functions $p \mapsto$ $\Phi_{i}\left(g, \lambda, \theta_{p}\right)(i=1,2)$ are exponentially convex and that for $\mu_{p, q}\left(g, \Phi_{i}, \Omega_{3}\right)$ the monotonicity property holds.

If $\Phi_{i}(i=1,2)$ are positive, then using Theorem 15 applied for $\varphi=\theta_{p} \in \Omega_{3}$ and $\psi=\theta_{q} \in \Omega_{3}$, it follows that

$$
\mathscr{M}_{p, q}\left(g, \Phi_{i}, \Omega_{3}\right)=-(\sqrt{p}+\sqrt{q}) \log \mu_{p, q}\left(g, \Phi_{i}, \Omega_{3}\right)
$$

satisfy

$$
\alpha \leq \mathscr{M}_{p, q}\left(g, \Phi_{i}, \Omega_{3}\right) \leq \beta, \quad \text { for } i=1,2 .
$$

If we set that the image of the function $g$ is $[\alpha, \beta]$, then we have that in this case $\mathscr{M}_{p, q}\left(g, \Phi_{i}, \Omega_{3}\right)$ are means (of the function $g$ ).

Example 4. Let

$$
\Omega_{4}=\left\{\phi_{p}: \mathbb{R}^{+} \longrightarrow \mathbb{R}^{+}: p \in \mathbb{R}^{+}\right\}
$$

be a family of functions defined by

$$
\phi_{p}(x)= \begin{cases}\frac{p^{-x}}{(\log p)^{2}}, & p \neq 1 ; \\ \frac{x^{2}}{2}, & p=1 .\end{cases}
$$

Since $\left(d^{2} / d x^{2}\right) \phi_{p}(x)=p^{-x}>0, \phi_{p}$ is convex function for $p>0$. From Remark 22 it follows that $p \mapsto\left(d^{2} / d x^{2}\right) \phi_{p}(x)$ is exponentially convex function, and from [10] we then also have that $p \mapsto\left[y_{0}, y_{1}, y_{2}\right] \phi_{p}$ is exponentially convex. Our family $\Omega_{4}$ of functions $\phi_{p}$ fulfills the condition given in Corollary 29. We assume again that interval $[\alpha, \beta]$ from our corollaries is a subset of $\mathbb{R}^{+}$, and so for our family of functions we have the following:

$$
\begin{aligned}
\mu_{p, q}\left(g, \Phi_{i}, \Omega_{4}\right) & \begin{cases}\left(\frac{\Phi_{i}\left(g, \lambda, \phi_{p}\right)}{\Phi_{i}\left(g, \lambda, \phi_{q}\right)}\right)^{1 /(p-q)}, & p \neq q ; \\
\exp \left(-\frac{\Phi_{i}\left(g, \lambda, i d \cdot \phi_{p}\right)}{p \Phi_{i}\left(g, \lambda, \phi_{p}\right)}-\frac{2}{p \log p}\right), & p=q \neq 1 ; \\
\exp \left(-\frac{2}{3} \frac{\Phi_{i}\left(g, \lambda, i d \cdot \phi_{1}\right)}{\Phi_{i}\left(g, \lambda, \phi_{1}\right)}\right), & p=q=1 .\end{cases}
\end{aligned}
$$

As before, we conclude that the functions $p \mapsto$ $\Phi_{i}\left(g, \lambda, \phi_{p}\right)(i=1,2)$ are exponentially convex, and for $\mu_{p, q}\left(g, \Phi_{i}, \Omega_{4}\right)$ the monotonicity property holds.

If $\Phi_{i}(i=1,2)$ are positive, then using Theorem 15 applied for $\varphi=\phi_{p} \in \Omega_{4}$ and $\psi=\phi_{q} \in \Omega_{4}$, it follows that

$$
\mathcal{N}_{p, q}\left(g, \Phi_{i}, \Omega_{4}\right)=-L(p, q) \log \mu_{p, q}\left(g, \Phi_{i}, \Omega_{4}\right),
$$

where $L(p, q)$ denotes the logarithmic mean defined by $L(p, q)=(p-q) /(\log p-\log q)$ for $p \neq q$, and $L(p, p)=$ $p$ satisfy

$$
\alpha \leq \mathcal{N}_{p, q}\left(g, \Phi_{i}, \Omega_{4}\right) \leq \beta, \quad \text { for } i=1,2 .
$$


If we set that the image of the function $g$ is $[\alpha, \beta]$, then we have that in this case $\mathcal{N}_{p, q}\left(g, \Phi_{i}, \Omega_{4}\right)$ are means (of the function $g$ ).

\section{Conflict of Interests}

The authors declare that there is no conflict of interests regarding the publication of this paper.

\section{References}

[1] D. S. Mitrinović, J. E. Pečarić, and A. M. Fink, "Uniform treatment of Jensen type inequalities," Mathematical Reports, vol. 16(66), no. 2, pp. 183-205, 1993.

[2] R. P. Boas, "The Jensen-Steffensen inequality," Publikacije Elektrotehničkog fakulteta Univerziteta u Beogradu, Serija Matematika, no. 302-319, pp. 1-8, 1970.

[3] J. E. Pečarić, F. Proschan, and Y. L. Tong, Convex Functions, Partial Orderings, and Statistical Applications, vol. 187 of Mathematics in Science and Engineering, Academic Press, Boston, Mass, USA, 1992.

[4] S. S. Dragomir, "Reverses of the Jensen inequality in terms of first derivative and applications," Acta Mathematica Vietnamica, vol. 38, no. 3, pp. 429-446, 2013.

[5] S. S. Dragomir, "Some reverses of the Jensen inequality with applications," Bulletin of the Australian Mathematical Society, vol. 87, no. 2, pp. 177-194, 2013.

[6] D. S. Mitrinović, J. E. Pečarić., and A. M. Fink, Classical and New Inequalities in Analysis, Kluwer Academic Publishers, 1993.

[7] B. Gavrea, J. Jakšetić, and J. Pečarić, "On a global upper bound for Jenssen's inequality," The ANZIAM Journal, vol. 50, no. 2, pp. 246-257, 2008.

[8] A. R. Khan, J. Pečarić, and M. Rodić Lipanović, "n-exponential convexity for Jensen-type inequalities," Journal of Mathematical Inequalities, vol. 7, no. 3, pp. 313-335, 2013.

[9] M. Klaričić Bakula, J. Pečarić, and J. Perić, "Extensions of the Hermite-Hadamard inequality with applications," Mathematical Inequalities and Applications, vol. 15, pp. 899-921, 2012.

[10] J. Jakšetić and J. Pečarić, "Exponential convexity method," Journal of Convex Analysis, vol. 20, no. 1, pp. 181-197, 2013. 


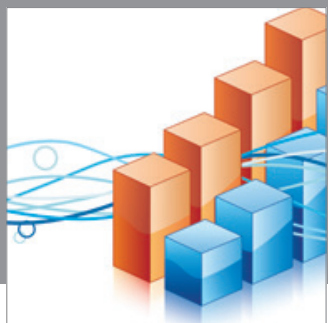

Advances in

Operations Research

mansans

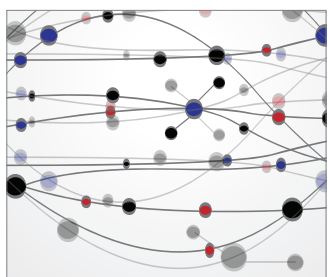

The Scientific World Journal
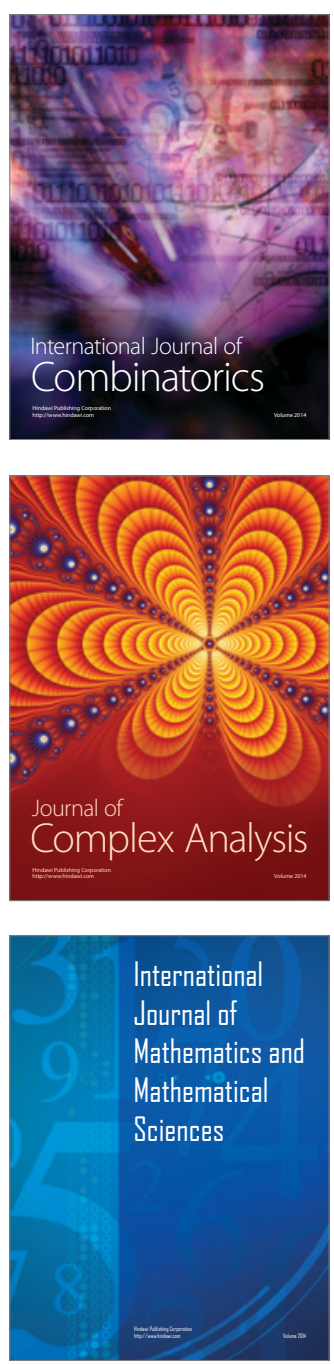
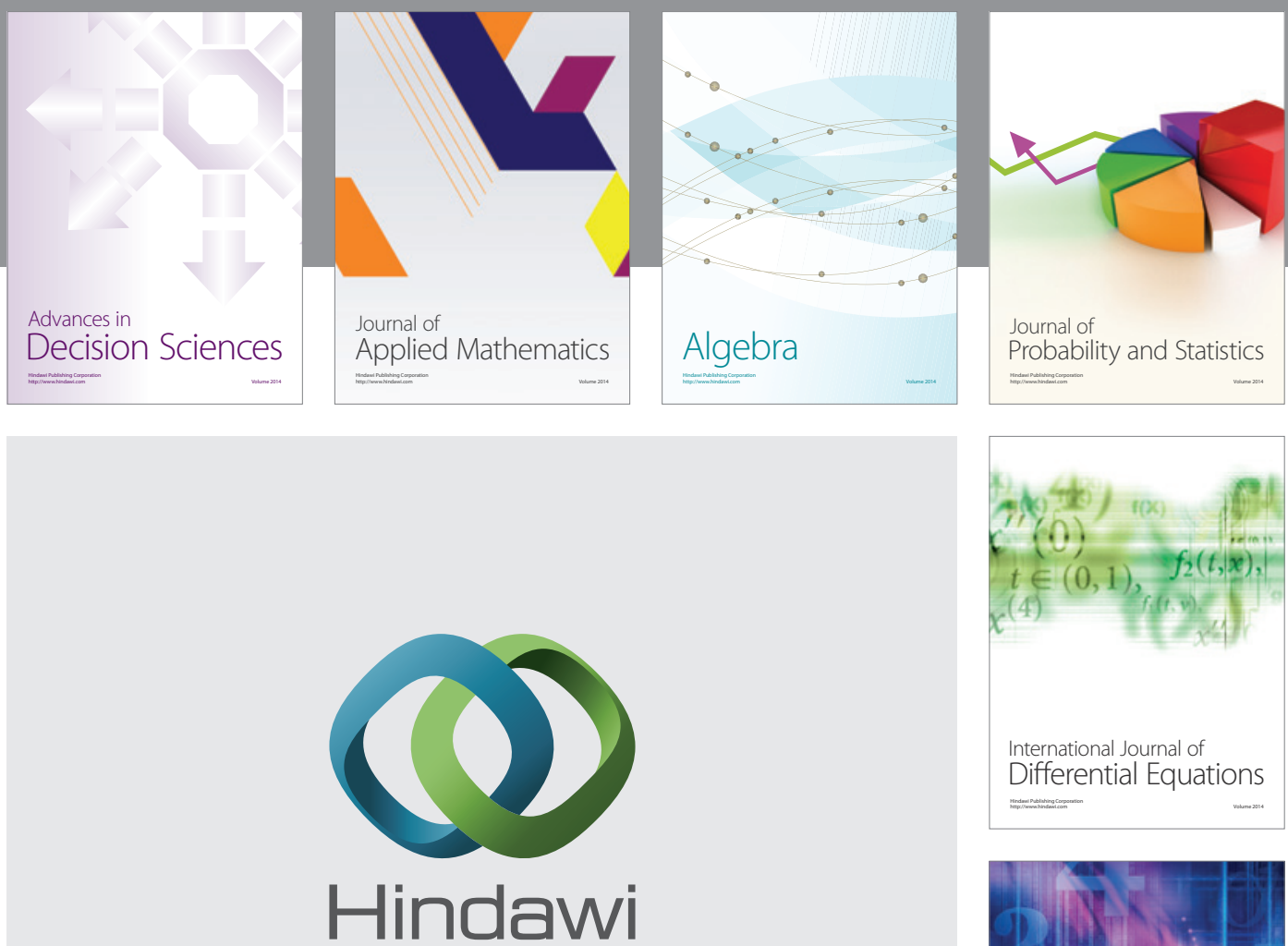

Submit your manuscripts at http://www.hindawi.com
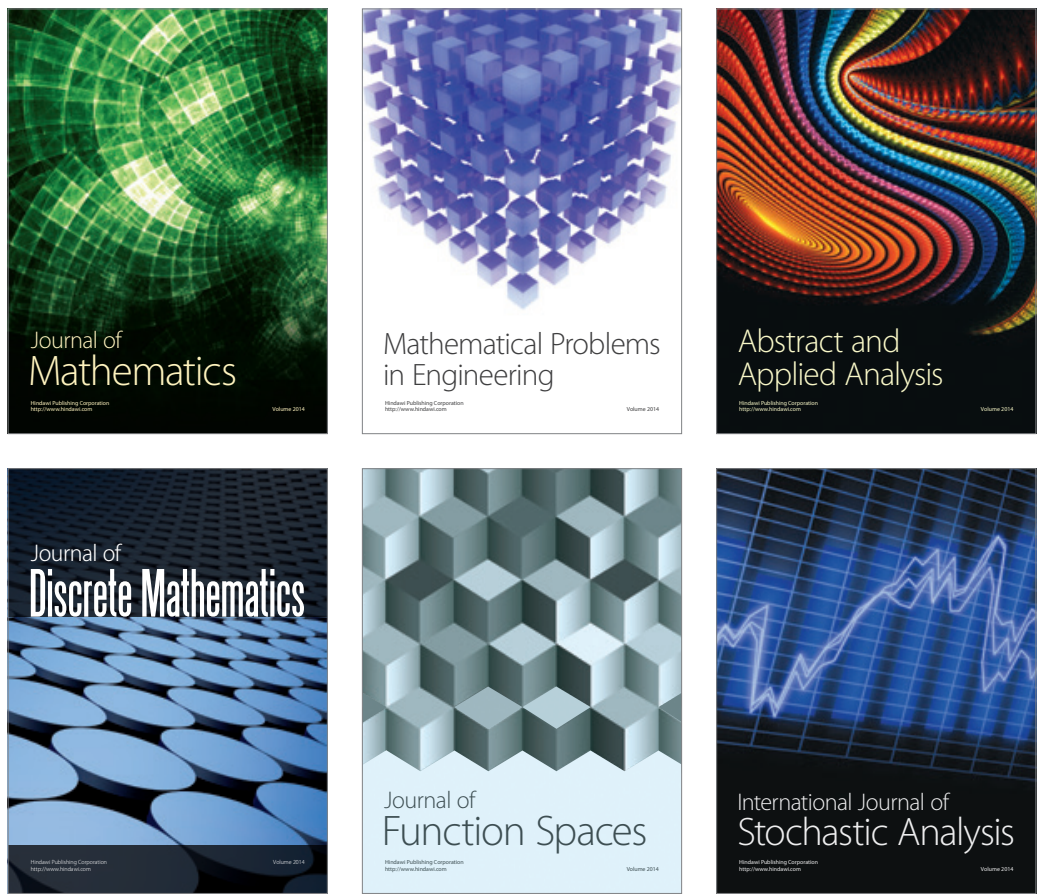

Journal of

Function Spaces

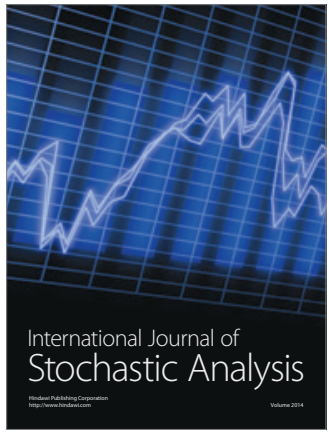

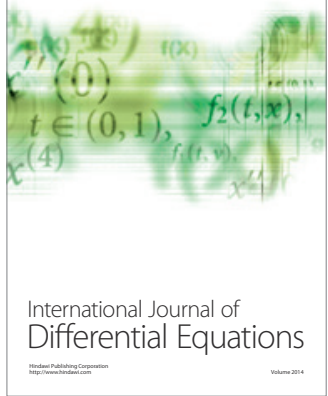
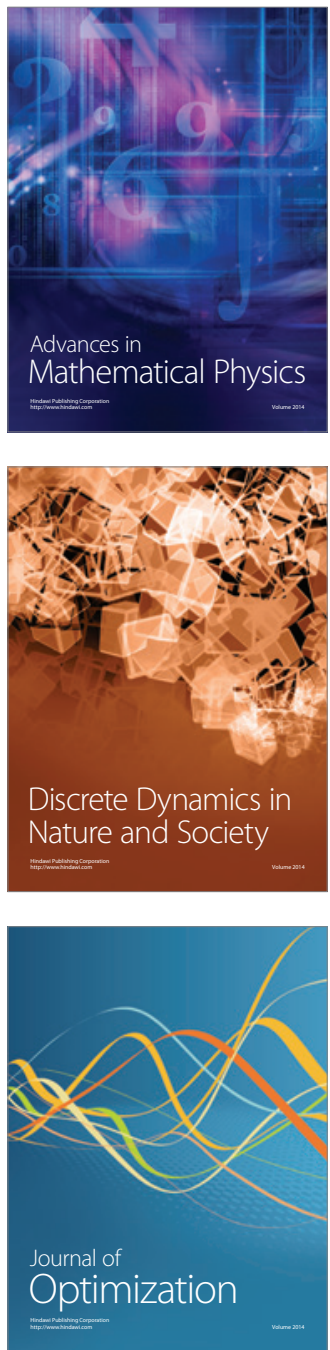